\title{
EL SISTEMA DE PARTIDOS EN EL SALVADOR (1982-1994)
}

\author{
Alvaro Artiga-González*
}

\section{Una importancia creciente}

F n lugares como América Latina, con la emergencia de proce Lsos de transición de regímenes políticos autoritarios hacia regimenes políticos democráticos, una de las preocupaciones teóricas y prácticas de los sujetos que impulsan tales procesos es la creación, desarrollo y consolidación de las instituciones propias de una democracia, y entre ellas el sistema de partidos políticos. No es que no existiesen partidos políticos durante la vigencia de los regímenes autoritarios. Es más, en algunos países como Chile lo que ocurrió fue una suspensión de la democracia política para dar paso a la dictadura militar. En estos casos se trata de la recomposición de instituciones democráticas pre-existentes.

En el caso salvadoreño, partidos políticos existen desde finales del siglo pasado aunque su naturaleza haya ido variando con el transcurso del tiempo desde ser partidos caudillistas hasta partidos de masas ${ }^{1}$. Pero, a excepción de 1931, es hasta 1994 donde participan partidos de todo el espectro político en un proceso electoral. $Y$ aún así, no se puede decir que esté consolidado el sistema de partidos. Considérese nada más los reacomodos internos, incluso a nivel ideológicos, que actualmente enfrentan todos los partidos después de las elecciones del pasado Marzo-Abril para darse cuenta de lo inapropiado del calificativo consolidado para el sistema de partidos en El Salvador.

- Prolesor / investigador del Depatamento de Sodologia y Clencas Pollticas de la UCA 
Seguros cada vez más de que la Revolución Socialista no tiene, por hoy, espacio ni internacional ni nacional y participando de la "ola democratizadora" de fin de siglo, se percibe la necesidad de fortalecer las estructuras partidarias para canalizar la lucha política. Esto ha hecho que a nivel teórico haya cobrado relevancia el tema del sistema de partidos. Ya existen los primeros estudios, aunque provisionales algunos, para los distintos paises de América Latina ${ }^{2}$. Para el caso específico de El Salvador hemos encontrado un trabajo que cubre el período 1982-1989. No es que con anterioridad no se hubiese estudiado a los partidos políticos en el país. Pero ciertamente no se lo habia hecho con las categorías con que ahora se estudia esa temática.

Sin entrar a un estudio a fondo del Sistema de Partidos salvadoreño, vamos a considerar aquellos elementos analíticos que nos arrojen luz en la explicación del resultado de las elecciones de 1994: La mayoritaria representación politica de derecha.

\section{Sobre el formato del sistema de partidos en El Salvador}

Das que Giovanni Sartori escribió su libro "Partidos y Siste mato (número de partidos) para hacer una tipología o una clasificación de los sistemas de partidos. Pero de alli en adelante se generó un debate alrededor de dicha tarea. Asi se han ido proponiendo nuevos criterios de clasificación y se ha modificado incluso la propuesta original del mismo Sartorit.

Cuando se debate sobre la conveniencia, o no, del esquema de Sartori para el análisis de los sistemas de partidos en América Latina se trae a cuenta el origen de dicho esquema: el análisis de sistemas parlamentarios. Por tanto, no podremos evitar caer en la polémica pertinente al estudiar el sistema de partidos en El Salvador si partimos de algunos planteamientos de Sartori. Aunque no es nuestra intención entrar en el debate teórico sino que aplicar algunas categorias, teniendo presente sus limitaciones, al caso salvadoreño.

Uno de los primeros problemas que se enfrentan al estudiar el sistema de partidos es el de la determinación del formato, es decir: ¿cuáles partidos se han de considerar?. Y es que el formato interesa en cuanto puede contribuir a determinar un conjunto de propiedades funcionales del sistema de partidos. Se avanza bastante, 
aunque no es suficiente, con solo determinar si estamos ante un monopartidismo, un bipartidismo o un multipartidismo. Cuanto mayor sea el número de partidos mayor será la complejidad del sistema de partidos. El problema radica en si deben tomarse en cuenta todos los partidos participantes en unas elecciones (aún en este caso se podrían dejar fuera partidos políticos que estando proscriptos influyen en la dinámica interpartidaria). Y si no se consideran todos los partidos, ¿cuál es el criterio para tomarlos en cuenta, cuál es la fuerza electoral que debe tener un partido para ser contabilizado? ${ }^{5}$

Para el caso salvadoreño el análisis se complica si tomamos en cuenta la existencia de tres tipos de elecciones: Presidencial, Diputados y Concejos Municipales. Si postulamos que el electorado vota por el partido más que por los candidatos en las elecciones de diputados y alcaldes, favorecida esta situación por la simultaneidad temporal de dichas elecciones, reducimos un tanto nuestro problema a la consideración de dos eventos electorales pero que sin embargo mantienen vigente el problema del formato. Veamos más de cerca el problema de la determinación del número de partidos a considerar. Vamos a partir de la hipótesis de que el régimen político salvadoreño entró en un proceso de reconstrucción a partir de 1982 con la elección de una Asamblea Constituyente y que poco a poco fueron estableciéndose los espacios necesarios para la no exclusión de ninguna fuerza política cuya culminación la constituyó el proceso electoral de 1994.

En el periodo que va desde 1982 hasta 1994 han habido siete procesos electorales (considerando como uno solo el de 1994):

1982: Asamblea Constituyente.

1984: Presidente y Vicepresidente.

1985: Asamblea Legislativa y Concejos Municipales.

1988: Asamblea Legislativa y Concejos Municipales.

1989: Presidente y Vicepresidente.

1991: Asamblea Legislativa y Concejos Municipales.

1994: Presidente y Vicepresidente.

1994: Asamblea Legislativa y Concejos Municipales.

En todo este período han participado 19 partidos políticos distribuidos según lo muestra el Cuadro No.1 (no se consideran las coaliciones de partidos para tener una mejor idea de la fragmentación del sistema y para que se perciba con mayor nitidez el 
problema de la determinación del formato).

En 1989, la Convergencia Democrática (CD) era una coalición formada por los partidos Movimiento Popular Social Cristiano (MPSC), el partido Social Demócrata (PSD) y el Movimiento Nacional Revolucionario (MNR). En 1994, CD era una partido resultado de la fusión del MPSC, el PSD y la Unión Democrática Nacionalista (UDN). Por otra parte, para la elección presidencial de 1994, CD, MNR y el FMLN participaron como coalición pero manteriendo las tres banderas y no bajo una sola en las papeletas de votación. Además, para 1989 el Partido Popular Salvadoreño (PPS), el Partido Liberación y el Partido Auténtico Institucional Salvadoreño (PAISA) se presentaron a la elección como la coalición Unidad Popular (UP).

\section{Cuadro No.1:}

Partidos políticos participantes en las elecciones presidenciales del periodo 1982-1994.

\begin{tabular}{|c|c|c|c|}
\hline PARTIDO & 1984 & 1989 & 1994 \\
\hline PDC & $x$ & $x$ & $x$ \\
\hline $\mathrm{AD}$ & $x$ & $\mathrm{x}$ & - \\
\hline POP & $x$ & - & - \\
\hline MERECEN & $\mathrm{x}$ & - & - \\
\hline PCN & $x$ & $x$ & $x$ \\
\hline PAISA & $\mathrm{x}$ & $\mathrm{x}$ & - \\
\hline PPS & $\mathrm{x}$ & $\mathrm{x}$ & - \\
\hline ARENA & $x$ & $\mathrm{x}$ & $x$ \\
\hline $\mathrm{CD}$ & - & - & $\mathrm{x}$ \\
\hline MPSC & - & $x$ & - \\
\hline PSD & - & $\mathrm{x}$ & - \\
\hline UDN & - & $x$ & - \\
\hline MNR & - & $\mathbf{x}$ & $x$ \\
\hline PAR & - & $\mathrm{x}$ & - \\
\hline LIBERACION & - & $x$ & - \\
\hline MAC & - & $x$ & $x$ \\
\hline MSN & - & - & $x$ \\
\hline MU & - & - & $\mathrm{x}$ \\
\hline FMLN & - & - & $x$ \\
\hline Total & 8 & 13 & 9 \\
\hline
\end{tabular}


El Cuadro No.2 nos muestra los partidos políticos que participaron en las elecciones parlamentarias en todo el período. De nuevo no se han considerado las coaliciones pre-electorales. Al respecto, en 1991 la CD era una coalición formada por el MNR, el MPSC y el PSD. En tanto que para 1994, hemos dicho que la CD era ya un partido resultado de la fusión del UDN, el MPSC y el PSD.

\section{Cuadro No.2:}

Partidos políticos participantes en elecciones para diputados en el periodo 1982-1994.

\begin{tabular}{|c|c|c|c|c|c|}
\hline PARTIDO & 1982 & 1985 & 1988 & 1991 & 1994 \\
\hline PDC & $\mathbf{x}$ & $\mathrm{x}$ & $x$ & $x$ & $\mathrm{x}$ \\
\hline $\mathrm{AD}$ & $x$ & $x$ & $x$ & $x$ & - \\
\hline POP & $x$ & $x$ & $\mathrm{x}$ & - & - \\
\hline $\mathrm{PCN}$ & $\mathrm{X}$ & $x$ & $x$ & $x$ & $x$ \\
\hline PPS & $\mathrm{x}$ & $x$ & - & - & - \\
\hline ARENA & $x$ & $\mathrm{x}$ & $\mathrm{x}$ & $\mathrm{x}$ & $x$ \\
\hline PAR & - & $x$ & $x$ & - & - \\
\hline MERECEN & - & $\mathrm{X}$ & - & - & - \\
\hline PAISA & - & $\mathbf{x}$ & $\mathrm{x}$ & - & - \\
\hline LIBERACION & - & - & $x$ & - & - \\
\hline$C D$ & - & - & - & - & $\mathrm{X}$ \\
\hline UDN & - & - & - & $x$ & 一 \\
\hline MNR & - & - & - & $x$ & $x$ \\
\hline MPSC & 一 & 一 & 一 & $\mathrm{x}$ & 一 \\
\hline PSD & - & 一 & - & $x$ & - \\
\hline MAC & - & - & - & $\mathrm{x}$ & $x$ \\
\hline MSN & - & - & - & - & $\mathrm{x}$ \\
\hline MU & - & - & - & - & $x$ \\
\hline FMLN & - & - & - & - & $\mathrm{x}$ \\
\hline Total & 6 & 9 & 8 & 9 & 9 \\
\hline
\end{tabular}

Una primera aproximación al formato del sistema de partidos nos dejaría con la impresión de una gran fragmentación. Estaría- 
mos tentados a decir que se trata de un pluralismo en la terminologia de Sartori6. Tenemos que adentrarnos en la discusión sobre los partidos que deben ser tomados en cuenta. Los datos que hemos traído a cuenta son predictivos, es decir, hacen referencia al momento pre-electoral, a los partidos que se inscribieron para participar en cada elección. Pero cuando se habla del formato suele considerarse los resultados electorales y, por tanto, se consideran los datos postdictivos. Refiriéndose a elecciones parlamentarias se han elaborado incluso indices para calcular el número de partidos teniendo en cuenta las proporciones de votos o de escaños alcanzados $^{7}$. El mismo Sartori daba las siguientes normas para contar:

a. Considerar solo los partidos que alcanzan escaños.

b. No contar aquellos que aún estando presentes en el parlamento no han participado en una mayoria de coalición. Es decir, contar los partidos que tienen un potencial para formar una coalición mayoritaria.

c. Contar a los partidos antisistema que, aunque no hayan formado una mayorfa de coalición, tienen una capacidad de veto como para alterar la dirección de la competencia, es decir, contar a los partidos con potencial de chantaje ${ }^{8}$.

El interés por determinar el número de partidos radica en su influencia sobre la conformación y la duración de un gabinete de gobierno. Así por ejemplo se ha asociado el bipartidismo con gabinetes monocolores y de mayor duración que los resultantes de un multipartidismo, lo que a su vez estaría generando una mayor estabilidad política. Pero el caso salvadoreño es diferente por el régimen presidencialista vigente. Con la elección del Presidente y Vicepresidente propuesto por un solo partido se garantiza la conformación de un gabinete monocolor. Y desde 1984, año de elección presidencial en el período que hemos denominado de reconstrucción del régimen político, los gabinetes han terminado su periodo constitucional de cinco años. Con lo que las razones señaladas que vuelven importante la consideración del formato del sistema de partidos en regimenes parlamentarios pierden validez para nuestro caso.

Por otra parte, la misma reglamentación electoral establece techos mínimos de votos alcanzados para evitar la anulación de cual- 
quier partido político y que explica la desaparición de siete de los diecinueve partidos participantes entre 1982 y 1994, al no haber alcanzado esa cantidad minima de votos 9

También se desprende del análisis de los datos sobre partidos participantes en elecciones que son pocos los partidos que han estado en todas las elecciones del periodo: PDC, PCN y ARENA. No se puede predecir con certeza qué será de los nuevos partidos: MSN, MU, CD y FMLN. Ni tampoco se puede asegurar que no habrán nuevos partidos en las elecciones de 1997. Recuérdese además que estamos considerando dos tipos de elecciones que, teniendo reglas de decisión diferentes, afectan las relaciones interpartidarias. Todos estos hechos obstaculizan la determinación del formato del sistema de partidos. Pero, por otra parte, refuerzan el planteamiento de que apenas se está en proceso de constitución del sistema partidario.

¿Podemos afirmar, al menos, que no estamos frente a una especie de bipartidismo histórico aunque esa fuese la sensación que nos transmiten los resultados electorales presidenciales desde la década del sesenta?

El que se hable de bipartidismo no significa necesariamente la existencia de solo dos partidos. Lo que importa es que un partido gobierna solo y se da la alternancia en el poder, aunque sea unicamente como expectativa o como posibilidad. Sartori señala las siguientes características del bipartidismo ${ }^{10}$ :

a) Dos partidos se hallan en condiciones de competir por la mayoría de escaños.

b) Uno de los dos partidos logra efectivamente conseguir una mayoria parlamentaria suficiente.

c) Este partido está dispuesto a gobernar solo.

d) La altemación o la rotación en el poder sigue siendo una expectativa creíble.

La necesidad de la mayoría de escaños queda planteada por el sistema parlamentario. Solo así se garantiza un gobierno unicolor. En los sistemas presidencialistas la mayoría parlamentaria busca más bien la no obstaculización de la gestión del partido en el gobierno. 
Pues bien, durante las décadas de los 60 y 70 la lucha partidaria por el gobierno giró alrededor de los partidos Demócrata Cristiano y de Conciliación Nacional. Pero la recurrencia al fraude electoral para anular el triunfo de la oposición partidaria aglutinada en la Unión Nacional Opositora (UNO)" en 1972 y 1977, nos lleva a considerar al sistema de partidos como semi-competitivo. El régimen político vigente no permitió realmente la alternancia en el poder. En estas circunstancias quedarían cuestionadas las elecciones mismas. En parte es una de las razones que favorecieron el desarrollo de las organizaciones político-militares que llegaron a constituir el FMLN y que optaron por la lucha militar como vía de acceso al poder del Estado.

Esa lucha constante entre dos partidos se repite en la década de los 80. Con la debilitación del ex-partido oficial (PCN), y el surgimiento del nuevo partido que aglutinaría a la derecha más poderosa (ARENA), pero en un contexto de guerra, la lucha política por la presidencia gira en torno a los partidos PDC y ARENA. Se reforzaría la imagen del bipartidismo si se toma en cuenta que por fin en 1984, el PDC logra el acceso al gobierno y en 1989 se da, por primera vez, la alternancia pacífica en el poder al ganar las elecciones el partido ARENA.

Hemos sostenido anteriormente la hipótesis de la reconstrucción del régimen político durante la década de los 80 . De acuerdo a esto, estamos en el proceso de constitución del sistema de partidos. Y si observamos más de cerca este proceso podemos arribar a una conclusión diferente de la del bipartidismo histórico. En primer lugar, el bipartidismo está asociado a la posibilidad de que uno de los partidos principales alcanze la mayoría electoral. En las tres elecciones presidenciales del período 82-94, dos veces ha sido necesaria una segunda vuelta, precisamente para "manufacturar" la mayoría. Curiosamente esto se produjo cuando el PDC alcanzó la presidencia en 1984 (en tiempos de guerra) y cuando ARENA vuelve a ganar (en tiempos de paz).

En el período han habido 5 elecciones de diputados. El cuadro No.3 nos muestra la distribución absoluta y porcentual de los escaños legislativos entre los distintos partidos.

Como podemos observar en dicho cuadro, unicamente en 1985 
un partido (el PDC) obtuvo la mayoria parlamentaria. Curiosamente, un año después de su triunfo en las elecciones presidenciales y el único proceso electoral en el que no fueron dados a conocer publicamente los resultados oficiales por parte del anterior Concejo Central de Elecciones. Podria pensarse que al igual que el año 1984 fue necesario "manufacturar" una mayoría para garantizar la gestión presidencial en tiempos de guerra y como parte de un proyecto contrainsurgente.

\section{Cuadro No.3:}

Distribución absoluta y porcentual de los escaños legislativos entre los partidos participantes en las elecciones para diputados en período 1982-1994.

\begin{tabular}{|l|c|c|c|c|c|}
\hline PARTIDO & 1982 & 1985 & 1988 & 1991 & 1994 \\
\hline ARENA & $19(31.7)$ & $13(21.6)$ & $30(50.0)$ & $39(46.4)$ & $39(46.4)$ \\
PDC & $24(40.0)$ & $33(55.0)$ & $23(38.3)$ & $26(31.0)$ & $18(21.4)$ \\
PCN & $14(23.3)$ & $12(20.0)$ & $7(11.7)$ & $9(10.7)$ & $4(4.8)$ \\
FMLN & - & - & - & - & $21(25.0)$ \\
CD & - & - & - & $8(9.5)$ & $1(1.2)$ \\
UNIDAD & - & - & - & - & $1(1.2)$ \\
MAC & - & - & - & $1(1.2)$ & - \\
PAISA & - & $1(1.7)$ & - & - & - \\
PPS & $1(1.7)$ & - & - & - & - \\
UDN & - & - & - & $1(1.2)$ & - \\
AD & $2(3.3)$ & $1(1.7)$ & - & - & - \\
\hline TOTAL & 60 & 60 & 60 & 84 & 84 \\
\hline
\end{tabular}

- EI MAC surgió de la fusión del partido MERECEN (Movimiento Estable Republicano Centrista) y del MADC (Movimiento Auténtico Demócrata Cristiano, escindido del PDC). Un grupo de diputados electos para el PDC se pasaron al MAC y asi, este nuevo partido contó con representación en la Asamblea y favoreció la mayoría de derecha con la frecuente alianza: MAC-ARENA-PCN. 


\section{Cuadro No.4:}

Resultados electorales para los tres partidos que obtuvieron más votos válidos en elecciones presidenciales en el período 1982-1994.

\begin{tabular}{|l|c|c|c|}
\hline PARTIDO & 1984 & 1989 & 1994 \\
\hline ARENA & 376,917 & 505,370 & 641,108 \\
& $(29.76)$ & $(53.82)$ & $(40.03)$ \\
PDC & 549,727 & 338,369 & 214,277 \\
& $(43.41)$ & $(36.03)$ & $(16.36)$ \\
PCN & 244,556 & 38,218 & 70,504 \\
& $(19.31)$ & $(4.07)$ & $(5.39)$ \\
FMLN-CD-MNR & - & - & 325,582 \\
& & & $(24.90)$ \\
\hline
\end{tabular}

Consecuentemente con lo arriba planteado, el debate parlamentario se ha basado en alianzas entre distintos partidos para lograr mayorías. Es interesante observar que desde 1988 estas mayorías parlamentarias han sido de derecha 0 , por lo menos, subordinadas a la gestión política del partido ARENA.

Por otra parte, si consideramos los resultados electorales que han obtenido los tres mayores partidos políticos en la disputa por la Presidencia y Vicepresidencia, tal como lo muestra el Cuadro No.4, lo que podemos postular al integrar el análisis hecho hasta aquí es una tendencia hacia la constitución de un sistema de partido predominante.

\section{Un pluralismo polarizado}

Rechazada la hipótesis del bipartidismo histórico, nos move 1 mos entonces en el espacio del multipartidismo. Esta conclusión se ve reforzada por el número de partidos, no solo participantes en las elecciones (siempre más de seis) si no partidos que obtuvieron escaños legislativos (a excepción de 1988, en el resto de elecciones parlamentarias obtuvieron escaños cinco o más partidos).

Ahora bien, la impresión del bipartidismo histórico tiene como fundamento la existencia de dos "polos" electorales. Hasta 1979 
existía un polo en la oposición y el partido gobernante constituía el otro polo.

No es imprescindible que los polos se ubiquen hacia los extremos de un "continuum" ideológico izquierda-derecha; lo que importa es que esos polos, aún estando hacia un mismo lado del "continuum" le imprimen la dinámica al sistema de partidos. Ellos determinan el sentido de la competencia política y electoral. $Y$ es precisamente en la coyuntura de una competencia electoral donde claramente se observan estos polos, al aglutinarse la mayor parte del electorado en torno a ellos.

En el periodo 1982-1991 el sistema de partidos sigue siendo bipolar. Pero hay que tener presentes los siguientes matices. En el terreno de la competencia electoral, ARENA y el PDC son los nuevos polos ante la crisis del PCN (partido gobernante en las décadas 60-70) que favorece el desarrollo de partidos como el mismo ARENA y PAISA. Los dos cuadros presentados anteriormente muestran el descenso experimentado en la fuerza electoral del PCN en este periodo.

Por otra parte, PDC y ARENA se repartieron juntos más del $70 \%$ de los escaños en todas las elecciones parlamentarias y más del $70 \%$ de los votos en las elecciones presidenciales. Nos interesa señalar en este momento las relaciones de oposición entre estos partidos. En el periodo considerado ambos controlaron, por lo menos una vez, el ejecutivo y el legislativo al mismo tiempo, con lo que la relación de oposición se presentó con una mayor claridad.

Ahora bien, mientras se va reconstruyendo el régimen político roto en 1979, otros sujetos sociales y políticos consolidan otro "polo", que ya venía desarrollándose durante la década de los 70, y que no participa en las elecciones de los ochenta al estar enfrentando política y militarmente al gobierno de turno. Nos referimos a todas las organizaciones sociales aglutinadas alrededor del FMLN. Aunque no se trataba de un partido político como los existentes en el plano formal de la política, su aspiración también era llegar al poder del Estado para desde allí impulsar su proyecto político.

En este contexto, se desarrolla otra relación de oposición, esta vez, entre el gobierno (y el partido alli) y el FMLN. De tal manera que realmente existen tres polos que le imprimen la dinámica al 
sistema de partidos en construcción. Los efectos que, sobre el sistema de partidos, tiene la acción política del FMLN se pueden ver claramente en la coyuntura de su "Propuesta para convertir las elecciones en una contribución a la paz", en Enero de 1989. El FMLN estaba dispuesto a incorporarse al proceso electoral apoyando la plataforma de la Convergencia Democrática, si se posponían por unos seis meses y se establecian condiciones mínimas para el libre juego democrático ${ }^{12}$.

Hablando de las características del Pluralismo Polarizado, Sartori señala:

a) La presencia de partidos antisistema importantes.

b) La existencia de oposiciones bilaterales.

c) Ubicación central de un partido o un grupo de partidos. Esto favorece la tendencia centrifuga.

d) Polarización ideológica.

e) Prevalencia de impulsos centrifugos sobre los centripetos que llevan a un debilitamiento del centro, manifestada en la pérdida persistente de votos en favor de uno de los extremos (o incluso de ambos).

f) Estructuración ideológica congénita: los partidos políticos (principales) están en desacuerdo acerca de los principios y las cuestiones fundamentales (doctrina).

g) Presencia de oposiciones irresponsables.

h) Una política de superoferta: cuando los partidos prometen irresponsablemente lo que no podrán cumplir ${ }^{13}$.

Aunque estas caracteristicas no se dan completamente en el plano formal de la política salvadoreña, ellas describen la dinámica prevaleciente en las relaciones interpartidarias en el periodo 1982-1991.

¿Qué pasa entre 1991 y 1994? Se produce la incorporación del tercer polo (el FMLN) a la lucha política formal. El sistema de partidos cuenta desde entonces con partidos políticos que cubren todo el espectro ideológico. Pero su incorporación no significa que la dinámica política del período anterior haya sido dejada atrás. Al contrario, los polos siguen siendo los mismos como lo demues- 
tran los resultados electorales de 1994 (ARENA, FMLN Y PDC se colocan como las tres primeras fuerzas políticas). El partido en el gobierno continua siendo un polo como antes, pero en la oposición encontramos dos polos. Esto nos conduce a no identificar polos con extremos, ni a postular que el polo que necesariamente esté en el centro implique que el partido allí sea de centro en el "continuum" ideológico. En todo caso se trata de un partido central por su ubicación en el espacio ideológico.

\section{Competencia centrifuga}

A 1 estudiar el tipo de competencia electoral es necesario acla presentar tendencias centripetas (es decir, hacia el centro). Anteriormente señalabamos que no había que confundir polos con extremos, ni la polarización como una tendencia hacia los extremos políticos. En el caso salvadoreño, hacia las ELECCIONES DEL SIGLO, el sistema de partidos se mostraba como tri-polar. ¿Qué tipo de competencia electoral pudo observarse?

La observación de ciertos fenómenos nos lleva a plantear la tendencia centrifuga:

a) la dificultad de lograr consensos entre los polos como consecuencia de las oposiciones bilaterales (FMLN-PDC y PDC-ARENA).

b) las alianzas partidarias hacia los polos en los extremos y no hacia el polo central (FMLN-CD-MNR y ARENA-MAC-PCN).

c) el debilitamiento del polo central y el fortalecimiento de los polos en los extremos (desde 1985, el PDC ha ido disminuyendo su fuerza electoral tanto en elecciones presidenciales como parlamentarias, al igual que su proporción de diputados. Desde el mismo año, ARENA fue aumentando su fuerza electoral y presencia en la asamblea legislativa. La posibilidad de mantener la guerra por parte del FMLN no puede explicarse totalmente si no se considera su base social que luego se expresaría como fuerza electoral en 1994).

d) la constante prédica del PDC asignándose una posición de centro ha buscado atraer apoyo desde los extremos más cercanos 
al centro. En estas condiciones, se puede decir que el polo central ha buscado su expansión hacia los polos en los extremos, "robándole" votos a dichos polos. Es un movimiento del centro hacia afuera, tipico de la tendencia centrifuga.

e) el continuado esfuerzo de los polos en los extremos para aparecer como polos distantes, diferentes. Esta situación podría haber favorecido un fortalecimiento del polo central (PDC) pero las condiciones particulares (de guerra) en que realiza su gestión gubernativa entre 1984 y 1989 pueden explicar su desgaste político y consiguiente debilitamiento.

f) como puede esperarse en una competencia centrífuga, los polos extremos fueron los que se ubicaron como primera y segunda fuerza política en las elecciones de 1994. Como resultado de la necesidad de una segunda vuelta en las elecciones presidenciales, los votos del polo central fueron disputados por los polos extremos, y de hecho parte del electorado central se tuvo que desplazar hacia uno de los extremos.

No vamos a entrar en este momento a la discusión sobre el origen, las causas, de la competencia centrifuga. Creemos que ellas rebasan el plano de la lucha política.

\section{El sistema de partidos y las elecciones del siglo}

R esumamos lo dicho hasta aquí: En el período 1982-1994, asisti Rmos en El Salvador a la construcción de un nuevo sistema de partidos que todavía no logra consolidarse, reflejo de que la transición política tampoco ha arribado a la democracia. Las caracteristicas que pueden aprehenderse de dicho sistema corresponden a las que en el esquema de Sartori son propias de un PLURALISMO POLARIZADO.

Sin haber hecho un traslado mecánico de la propuesta de Sartori, hemos analizado los puntos referentes al formato, la polarización y la competencia electoral. Hemos llegado a la conclusión de que estamos ante un sistema pluripartidista que presenta tres polos y una competencia de tipo centrífuga.

Señalamos en su oportunidad el tradicional choque entre dos polos ( uno en el partido gobernante y el otro en la oposición). Las condiciones particulares por las que atravesó El Salvador llevaron 
a la formación de un tercer polo (segundo en la oposición). Esta situación nos permite hablar de una oposición fragmentada en polos. Esto es observable en distintos momentos del periodo estudiado en este trabajo, de la siguiente manera:

1982-1988: Polo en el Gobiemo o en PDC

la Asamblea:

Polos en la oposición:

(en la política formal)

ARENA

(en la guerra)

FMLN

1988-1991: Polo en el Gobierno o en

ARENA

la Asamblea:

Polo en la oposición:

(en la política formal)

PDC

(en la guerra)

FMLN

1994:

Polo en el Gobierno o en la Asamblea:

ARENA

Polos en la oposición:

FMLN y PDC.

Leído de esta manera el sistema de partidos en El Salvador, podemos postular que la competencia centrifuga y la fragmentación en polos en la oposición favorecerían la concentración de votos en el polo en el gobierno (el partido ARENA). De mantenerse este tipo de competencia electoral y fragmentación en polos en la oposición, el sistema de partidos puede irse consolidando como un sistema de partido predominante ${ }^{14}$. 
1. Hernández Turcios, Héctor (1978). "Régimen de partidos políticos en El Salvador", pp.69-70. Guatemala: Editorial I.N.C.E.P.

2. Por ejemplo el esfuerzo realizado por Nohlen, Dieter (1993) "Elecciones y Sistemas de Partidos en Américn Latina", publicado por el Instituto Interamericano de Derechos Humanos.

3. Cfr. Córdova-Macias, Ricardo (1992). "Procesos electorales y sistemas de partidos en El Snlvador (1982-1989)", Documento de Trabajo publicado por la Fundación Dr. Guillermo Manuel Ungo.

4. Cfr. Sartori, Giovanni (1980). "Partidos y sistemas de partidos, (primera parte)", Madrid: Alianza Editorial S.A.

5. Lijphart, Arend (1987). "Los democracins contemparaneas", p.131. Barcelona: Editorial Ariel, S.A.

6. Sartori, Giovanni. Op cit, pp.162-163.

7. Lijphart, Arend. Op cit, p.135.

8. Sartori, Giovanni. Op cit, Pp.154-158.

9. El actual Código Electoral establece que serán anulados los partidos que no alcanzen un $1 \%$ de los votos válidos en la elección en que participan.

10. Sartori, Giovanni. Op Cit.

11. Formada por los partidos PDC, MNR y UDN, con preeminencia del PDC, con lo que se refuerza la imagen bipartidista del sistema.

12. Cfr. Revista ECA \#483-484, Enero-Febrero de 1989. San Salvador: Universidad Centroamericana "José Simeón Cañas".

13. Sartori, Giovanni. Op Cit, pp.165ss.

14. Las conclusiones explicativas del resultado electoral de 1994 señaladas en este trabajo son parciales en cuanto forman parte de un trabajo más amplio que incluye: el sistema de partidos, el sistema electoral, el imaginario del votante y las anormalidades e irregularidades del proceso electoral de 1994. 